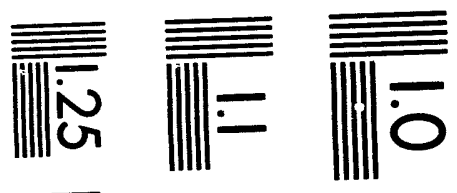

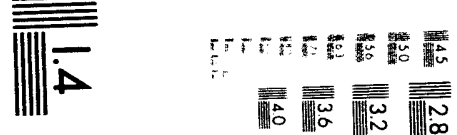

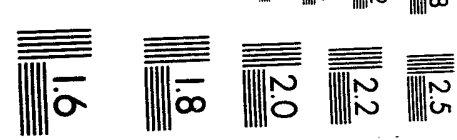



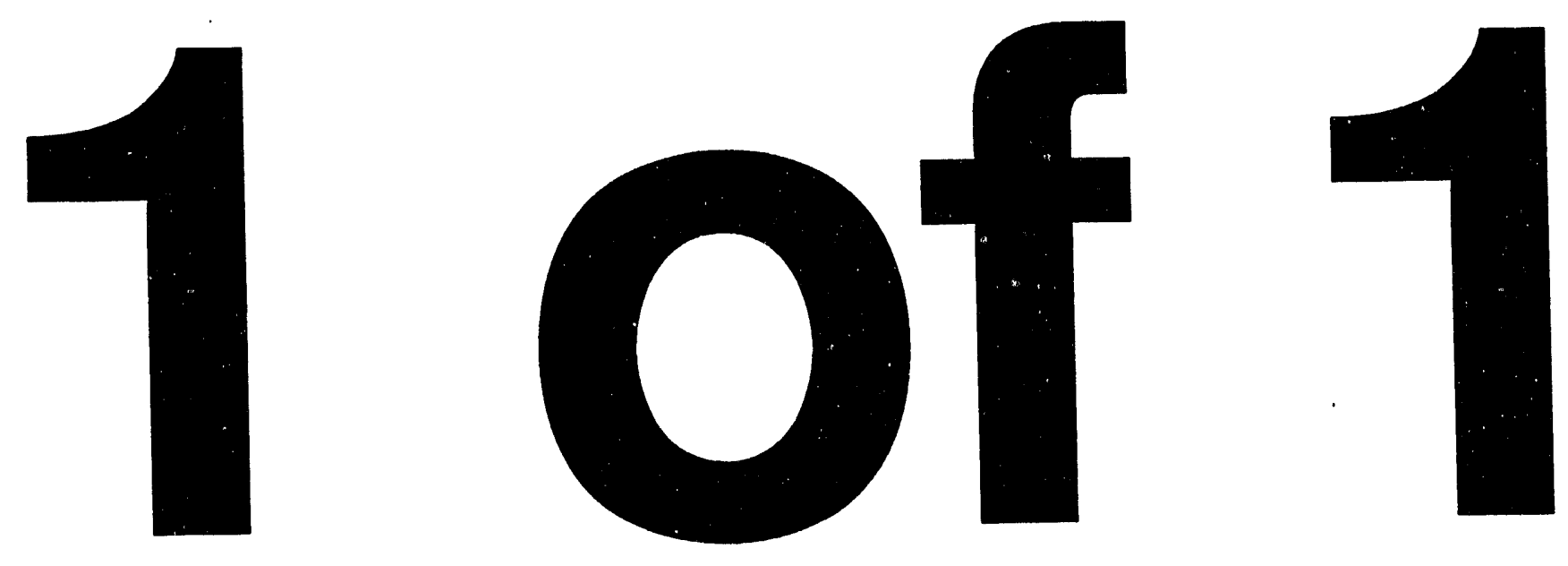
TITLE: ADAPTIVE OPTIMIZATION AND CONTROL USING NEURAL NETWORKS

AUTHOR(S):

William C. Mead, Stanley K. Brown, Roger D. Jones, P. Stuart Bowling, Christopher W. Barnes

SUBMITTED to: Proceedings of the ICALEPCS'93

\section{DISCLAIMER}

This report was prepared as an account of work sponsored by an agency of the United States Government. Neither the United States Government nor any agency thereof, nor any of their employees, makes any warranty, express or implied, or assumes any legal liability or responsibility for the accuracy, completeness, or usefulness of any information, apparatus, product, or process disclosed, or represents that its use would not infringe privately owned rights. Reference herein to any specific commercial product, process, or service by trade name, trademark, manufacturer, or otherwise does not necessarily constitute or imply its endorsement, recommendation, or favoring by the United States Government or any agency thereof. The views and opinions of authors expressed herein do not necessarily state or reflect those of the United States Government or any agency thereof. 


\title{
Adaptive Optimization and Control Using Neural Networks
}

\author{
W. C. Mead, S. K. Brown, R. D. Jones, P. S. Bowling, and C. W. Barnes \\ Los Alamos National Laboratory \\ October 22, 1993
}

\begin{abstract}
Recent work has demonstrated the ability of neural-network-based controllers to optimize and control machires with complex, non-linear, relatively unknown control spaces. We present a brief overview of neural networks via a taxonomy illustrating some capabilities of different kinds of neural networks. We present some successful control examples, particularly the optimization and control of a small-angle negative ion source.
\end{abstract}

\section{Introduction}

This paper presents an overview of Neural Networks (NN) and control-related NN applications. ${ }^{1-4}$ Artificial neural networks offer many benefits for prediction and control applications. They learn inductively from data, thus requiring little problem-specific knowledge. They learn on-line, so they can predict behavior of drifting systems. They can be implemented in software, which is relatively inexpensive to port to different computers or applications, or in hardware, which enables fast real-time operation.

We present a brief taxonomy, and discuss a few of the basic NN architectures. We illustrate NN capabilities by giving a number of "toy" and "real-world" control applications.

We discuss CTL, 5 an adaptive, NN-based controller for a small-angle, surfaceplasma negative ion source. 6 CTL performs a machine optimization and control task that had, before this work, only been successfully performed by trained human operators, and which had resisted two prior attempts at automation, one using expert systems technology and another using modern control theory. 7

The paper is organized as follows. Part I provides an overview of neural networks. Part II presents some typical NN architectures and applications, and a few application hints and tips. In part III, we discuss the negative ion source control problem, CTL adaptive controller concepts, and CTL tests and performance.

\section{Overview of Neural Networks}

An artificial neural network (NN) consists of three important compon ents, i, indicated schematically in Fig. 1: basis functions, network connectivity or arilit iure, and learning or training algorithms. Patterned loosely after biological sysien s, the bisis functions provide a set of identical, simple processing elements with a non ear inpl: output transfer (or activation) function. The network connectivity or architer iurs! determines the way information is distributed, transmitted, or stored by th netwicks Depending upon the network, the basis functions and/or the connections n sses. "weights" that are adjusted during training to permit the neural network in "lem ${ }^{2}$ " 4 , desired function. The learning algorithms are generally iterative expressis 6, adicoting the weights of the NN, based on the presentation of members of a training sivig vectors of input/output exampies that express the desired mapping behavior. inural network architectures include an input layer that passes all inputs to nodes (basis functions) in the first hidden layer, one or more hidden layers that are not directly connected to the outside world, and an output layer that combines outputs from the last hidden layer into the prescribed output configuration. 
The field of neural computing has benefited from multidisciplinary knowledge in fields as diverse as behaviorism, biology, engineering, mathematics, neurology, and physics. In return, it is hoped, neural networks have benefited those fields through theoretical and computational insights and applications.

Over the past 20 years, numerous researchers and extensive applications' needs have created an impressive variety of neural networks. Only some of the known NN's are indicated in the taxonomy of Fig. 2. By now, the NN universe has reached nearly uncountable proportions, so that only a dedicated (artificial) zoologist could catalog them! The taxonomy shown groups the networks by six general architectural types, a grouping that also reflects rough network performance characteristics. Networks in the upper left of Fig. 2 are those that serve most frequently in the field of dynamical system modeling, optimization, and control, so these will be emphasized here.

Neural networks have proven successful in many applications areas. These include ${ }^{1-4}$ dynamical system modeling, optimization, control, signal processing, data compression, image processing, feature location and extraction, fault detection and diagnosis, system or object classification, behavior capture, handwriting and speech recognition, knowledge extraction and representation, and machine learning.

\section{A Few Neural Networks and Application Examples}

Backpropagation Network. Properly referred to as the Feedforward-Backpropagation network, but popularly nicknamed the Backpropagation (BP) network, this neural net is one of the workhorses of the field. ${ }^{8}$ The BP basis function is the sigmoid, illustrated in Fig. 3. The network architecture is fully connected, as indicated in Fig. 1, with an input layer that feeds all inputs to all neurons or basis functions in the first hidden layer. Since the basis function is so simple, BP nets often must employ two or more hidden layers.

The training algorithm is widely published, $1-4,8$ and consists of backpropagation of errors, using the derivative of the basis function. Although widely used, the BP network has numerical difficulties that make it inefficient or ineffective in some cases: long training times and poor training convergence (training paralysis, local minima, and susceptibility to overtraining).

In spite of its numerical limitations, the BP net has successfully solved a number of difficult control problems. Two non-trivial examples are presented, next. Note that in both these examples, an off-line simulation model of the system to be controlled plays an important role in training the neural networks, providing low cost training data and lowrisk operation while the network makes errors during learning.

Truck Backer-Upper. Most recently, the famous BP-net "truck backer-upper" has been extended to back a double tractor-trailer truck. Usually, this application 3,9 is solved using two BP NN's: one to represent the truck dynamics, and one to control the steering. The time-lapse illustration in ref. 3 (p. 211) shows the double trailer truck being driven from its initial position to its final destination, (approximately) normal to a loading dock. The control network had 5 inputs, 25 hidden nodes, and 1 output. The training of this network was performed incrementally using successively more difficult test examples. It took about 2000 training runs to reach a successful control capability.

Freeway Automobile Driver. This example illustrates the splitting of a complex control problem into two parts, each of which is solved by a separate BP network. The task is to control the speed, steering, and lane-change decision-making for a particular vehicle driving among other cars on a two-lane freeway. 2,10 One network is assigned the task of accepting 7 inputs specifying the distances and speeds to neighboring vehicles and producing two outputs: one controlling the car's own speed, the other deciding when to issue a lane-change command. The second network accepts car position and orientation 
information and controls the car's steering. This network was trained by recording data generated while human "drivers" operated a computer driving simulation. The networks' adherence to the behaviors of the training driver is apparently quite striking. Obviously, one should use discretion in choice of the "model" driver!

Radial Basis Function Net. The Radial Basis Function (RBF) network replaces the sigmoid basis function by a gaussian. $1-4,11$ With this slightly more complicated basis function, some of the BP-net's most serious numerical problems are avoided. For the $\mathrm{RBF}$, a single hidden layer suffices. The training algorithm becomes linear and free of local minima. The one penalty paid is that, depending upon the problem, the RBF network can require substantially larger training sets than does backpropagation.

Connectionist Normalized Local Spline Net. The Connectionist Normalized Local Spline (CNLS) net $5,12,13$ is a variant of the RBF network that retains the lower-datarequirement of the BP net. By using normalized gaussian basis functions, which are broadened in a training-set-dependent way, plus a linear "correction" term, the CNLS net achieves the lower training data requirement and better generalization capabilities of the BP network. At the same time, it remains a single-hidden-layer network, with fast, linear training algorithms, like the RBF.

Hints and Tips for Neural Network Solutions. Before discussing some specific applications of CNLS-net, we mention a few numerical aspects of neural network solutions that apply, to a greater or lesser extent, to most neural networks.

A crucial ingredient to the success of any neural network solution is an adequate representation for the problem at hand. Representation is the term that describes the preand post-processing performed to determine the content and meaning of the neural network inputs and output(s). The representation chosen should be compact, lowdimensional, and information-rich. At one extreme is a representation that presents the network with numerous, low-information-density inputs. This presents the $\mathrm{NN}$ with a needle-in-the-haystack problem, and can easily confuse the network or require an impractically large training set. At the other extreme is a representation that does not contain the information required to solve the problem. In this case, the network is reduced to the role of a fortune teller, a role that NN's can play, but not a desirable one.

A second ingredient to many successful NN-based solutions is incorporation of problem-specific knowledge. Although not always necessary, using problem-specific knowledge can help to achieve a low-dimensional, compact representation. Several effective ways of incorporating such knowledge exist. Examples include formulating a cost or energy function that directly influences the NN's training algorithms; discretizing, limiting, and/or subdividing the problem's parameter space; or building applicable physical models into the data pre- or post-processing surrounding the network.

Certain training aids can help to ensure rapid, efficient learning conditions. These include: tumbling the input training vectors (changing the presentation sequence on successive presentations to the network); replication of rare (but important) training vectors; and performing competitive or complementary solutions using multiple networks (perhaps combined with voting or weighting of the network outputs).

Finally, as will be shown in the CNLS-net examples, two aspects of a problem's parameter space can suggest the utility of other numerical techniques. A parameter space with sharp cliffs, and perhaps hysteresis, suggests the use of recurrence: feeding one or more network outputs back to NN inputs. Input data which is noisy due to instrumental or other causes can suggest the use of Kalman filtering: in effect, automatically weighting the training vectors inversely by their frequency to accomplish averaging wherever possible. 
Modeling the Free Electron Laser's Control Space. Figure 4 shows a portion of the control space of a Free Electron Laser, as modeled by CNLS-net with recurrence, and the corresponding error surface. Using feedback, the network with gaussian basis functions can model a steep cliff.

Predicting and "Controlling" the Tides of Venice. The city of Venice has been gradually sinking. 14 Normal tides do not pose a great threat to the city's architecture and art, but unusual tides associated with storms could cause serious damage. The city uses a warning siren to initiate protective measures and a gate at the entrance to the lagoon to restrict tidal surges. The gate cannot be closed needlessly because it interrupts commerce and traps pollution. A local committee of observers records the tidal data and predicts when a dangerous surge is about to occur.

We have trained CNLS-net (including Kalman filtering) on the data from 1984, and ubtained the predictions shown in Fig. 5 for 1985 data. CNLS-net, with 12 hidden nodes, accepts tidal data from the previous 12 hours and predicts between one hour into the future (with RMS error of about 1\%) and 12 hours into the future (with RMS error about 5\%). 14 The plot shows 3-hour predictions.

\section{Optimization and Control of a Small-angle Negative Ion Source}

The Negative Ion Source Control Problem. At the Discharge Test Stand (DTS), Los Alamos National Laboratory is developing and automating a high-brightness negative ion source for various accelerator applications. From a control viewpoint, the negative ion source is a black box with four main control "knobs": the anode and cathode heater temperatures, the arc supply voltage, and the hydrogen gas flow rate. The behavior of the ion source is characterized by several diagnostics. Scalar readouts of the control variables and digitized waveforms of the arc voltage and current and the beam current indicate machine status. The success of the entire control operation is determined by the production of a "good" beam-current waveform: we seek to obtain high amplitude, low noise, and high pulse-to-pulse repeatability.

The negative ion source is driven by repetitive discharge of a $3 \mathrm{mf}, 400-600 \mathrm{~V}$ capacitor bank. The switched discharge pulse is $0.6-\mathrm{ms}$ duration, repeating every $0.2 \mathrm{~s}$, at typical arc currents of 150 amps. Instantaneous arc power levels are $20-50 \mathrm{~kW}$.

The negative ion source is compact and has a small active region, Fig. 6.15 The gap hetween the anode and cathode is less than $1 \mathrm{~mm}$ in extent. Much physics and chemistry occur in this small plasma source. When the anode is heated, a cesium vapor evolves from a powder-packed cavity, coating the cathode surfaces and reducing its work function. An applied magnetic field forces electrons to spiral, enhancing the production of negative ions in the hydrogen plasma. Negative ions are extracted by a biased grid structure into a small, high-brightness beam.

Diagnostic waveforms indicate ion source operational conditions, but with very indirect mapping to required control actions. Arc voltage and current, and beam current waveforms show the occurrence of various instabilities and non-steady source operating conditions.

The ion source control space is very complicated. Maps ${ }^{7}$ of beam current, which we want to maximize, and beam noise, which we want to minimize, as a function of hydrogen gas flow rate and arc supply voltage, and for three values of the anode temperature setting, show part of the control challenge. The contours in each twodimensional map are themselves complicated, with devious derivatives and multiple peaks and valleys. The shape of the operating landscape shows strong dependence on changes in the anode temperature and there are also significant differences between th: settings required to maximize current and those necessary to minimize noise. Now, 
imagine what happens in the fourth important control variable, the cathode temperature, and in the third beam quality measure, the pulse-to-pulse repeatability. We seek an overall optimum in terms of three beam quality characteristics as a function of four sensitive control variables. Finally, add the fact that source operation and maintenance variations lead to significant nonreproducibility from one mapping of control space to the next. By now the control challenge posed by this ion source should be quite clear!

Adaptive Controller Concepts. CTL is organized as indicated in the schematic shown in Fig. 7. The machine interface included regulating loops to decouple the control variables. The CTL supervisor and operator interface permitted human intervention, if required, and determined the overall sequence of CTL's operation. The instinct module governed data point acquisition and sparse multiparameter scanning. It provided pointsetlling logic and a discretized, bounded operating space. A figure of merit was constructed with terms proportional to beam current amplitude, the beam noise, and the pulse-to-pulse repeatability. The current operational database was used to train the neural network, CNLS-net. The tune-up of the source consisted of iterations in which the current neural net prediction of the optimum operating point was tested on the machine, the new datapoint was added to the training database, the neural network was retrained, and a new prediction of best operating point was obtained. The optimization scheme was open ended, adjustable, incomplete, and adaptively phased. Initial development of CTL was facilitated by off-line testing using a simple physics-based model of the negative ion source.

The artificial neural network, CNLS-net, 12,13 discussed above, provided excellent performance as an adaptive plant model in this application. The four machine control settings are inputs to the network. The network output provides a prediction of the source's performance, as indicated by the figure of merit, over the entire control space.

Adaptive Controller Performance. CTL has controlled the DTS ion source for several scan and control runs, with good results to date. Sparse multiparameter scans provided the first systematic observations of the changes in ion source parameter space. CTL tuned the source from first arc to optimum performance in six "cold" tune-ups, averaging 2.5 hours each. After short interruptions due to system "crashes," CTL retuned the source in ahout 15 minutes. Finally, CTL maintained the source near optimum for 5 holding runs, totaling about 5 hours, with no signs, so far, of serious limits.

The history of the fourth CTL control run, Fig. 8, shows optimization from "cold" startup in about 2 hours, rapid "warm" restarts, and good beam maintenance over about 3 hours. During the tune-up, the neural network predictions are initially quite inaccurate. Similarly, the early part of the gradient ascent examines some control settings that turn out to be poor. Nonetheless, the best known operating point chosen when the searchconvergence criterion is satisfied is a good one, and CTL maintains this good beam quality quite well.

As indicated by the wide variation in the final control setpoints, Table 1 , the negative ion source exhibits very complicated behavior. However, CTL appears capable of obtaining consistent performance, as indicated by the uniformly good final figure of merit. Not only is the figure of merit achieved from run-to-run relatively consistent, but so are the beam waveforms. The six low-impedance runs led to quite similar beam quality, superimposed in Fig. 9, and provided beam quality that is comparable with that achieved by experienced human operators.

CNLS-net played an important role in the optimizations. Fig. 10 displays averaged running statistics for the four CTL optimization phases. The two neuralnetwork optimization phases (NJ and NJS) used less than half the operating time, while providing some $80 \%$ of the total figure of merit improvement. 


\section{Summary and Conclusion}

We have presented a number of neural network architectures and applications to control problems. We expect that this general area will remain fertile for the foreseeable future, as the values and successes of adaptive control continue to lead to new technological capabilities.

\section{Acknowledgements}

This work has benefited from the contributions of many students and colleagues. In addition, we are grateful for widespread support and assistance from Los Alamos management and staff in the Applied Theoretical Division and the Accelerator Technology Division. This work was supported in part by the U.S. D.O.E. under the Los Alamos Laboratory Directed Reasearch and Development (LDRD) program. The Discharge Test Stand instrumentation and small-angle negative ion source were built for the Neutral Particle Beam program, supported by the U.S. Army Strategic Defense Command.

\section{References}

1Philip D. Wasserman, Neural Computing Theory and Practice (Van Nostrand Reinhold, New York, NY, 1989).

2Robert Hecht-Nielsen, Neurocomputing, (Addison-Wesley, NY, 1989).

${ }^{3}$ Alianna Maren, Craig Harston, and Robert Pap, Handbook of Neural Computing Applications, (Academic Press, San Diego, CA, 1990).

\section{${ }^{4}$ W. Thomas Miller, III, Richard S. Sutton, and Paul J. Werbos, Neural Networks for} Control, (MIT Press, Cambridge, MA, 1991).

5W. C. Mead, P. S. Bowling, S. K. Brown, R. D. Jones, et al., "Optimization and control of a small-angle negative ion source using an on-line adaptive controller based on the connectionist normalized local spline neural network," Nucl. Instr. Meth., B, 271 (1992).

6Yu. I. Belchenko, G. I. Dimov, and V. G. Dudnikov, Nucl. Fusion 14, 113 (1974); H. V. Smith, J. D. Sherman, and P. Allison, "Pulsed $\mathrm{H}^{-}$beams from Penning SPS sources equipped with circular emitters," Los Alamos National Laboratory Rpt. no. LA-UR-883290 (1988).

7J. A. Johnson, R. S. Biddle, H. D. Holt, S. K. Brown, and P. S. Bowling, "Status of automatic control of the ATS ion source," Los Alamos National Laboratory, AT-2 Tech. Note no. 89-03 (1989); J. A. Howell, C. W. Barnes, S. K. Brown, G. W. Flake, et al., Nucl. Instr. and Meth. A293, 517 (1990).

8P. Werbos, "Beyond regression: new tools for prediction and analysis in the behavioral sciences," PhD Dissertaion, (Harvard Univ., Cambridge, MA, 1974); D. B. Parker, "Learning Logic," Invention Rpt. S81-64, File 1, Office of Techn. Licensing, (Stanford Univ., Stanford, CA, 1982); D. E. Rumelhart, G. E. Hinton, and R. J. Williams, "Learning internal representations by error propagation," in Parallel Distributed Processing, I, (MiT Press, Cambridge, MA, 1986), 318. 
9D. Nguyen and B. Widrow, in Proc. First Int'l Joint Conf. on Neural Networks, II, (IEEE Press, NY, 1989), 2.57.

10J. F. Shepanski, Proc. of the Int. Conf. on Neural Networks, I, (IEEE Press, NY, 1988), 465.

$11 \mathrm{~J}$. Moody and C. J. Darken, "Fast learning in networks of locally-tuned processing units," Neural Comp. 1, 281 (1989).

${ }^{12}$ R. D. Jones, Y. C. Lee, C. W. Barnes, G. W. Flake, K. Lee, P. S. Lewis, and S. Qian, "Function approximation and time series prediction with neural networks," Los Alamos National Laboratory Rpt. no. LA-UR-90-21 (1990) and Proc. Joint Conf. on Neural Networks, I, San Diego, CA, June 17-21, 1990, (IEEE Press, NY, 1990), 649.

${ }^{13}$ R. D. Jones, Y. C. Lee, S. Qian, C. W. Barnes, et al., "Nonlinear adaptive networks: A little theory, a few applications," Los Alamos National Laboratory Rpt. no. LA-UR-91273 (1991) and Proc. First Los Alamos Workshop on Cognitive Modeling in System Control: Theoretical Foundations and Prospects for Applications, Santa Fe, NM, June 10-14, 1990, (Addison Wesley, NY, 1990).

14I. Poli and R. D. Jones, "A Neural Net for Predicting Noisy Data," Los Alamos National Laboratory Rpt. no. LA-UR-91-671 (1991).

15J. A. Yankeelov, III, "Study of a self-contained arc discharge current modulator," M.S. thesis, Univ. of New Mexico (1990). 


\section{Tables}

Table 1. Summary of the six low-impedance and one high-impedance CTL control runs. Final control setpoint (indicated in the discrete operating space) varies widely, but CTL obtains consistently good final figure of merit.

$\begin{array}{cccc}\text { Run } & \text { Einal Control Setpoint } & \text { Impedance } & \text { Einal Fig"re of Merit } \\ \text { MN-MO } & 6,6,4,1 & \text { low } & 0.692 \\ \text { MP-MQ } & 6,6,3,3 & \text { low } & 0.691 \\ \text { MR-MS } & 4,4,6,4 & \text { low } & 0.691 \\ \text { MT-MW } & 6,1,3,3 & \text { low } & 0.696 \\ \text { NA } & 6,1,3,4 & \text { low } & 0.694 \\ \text { NU-NV } & 1,1,3,4 & \text { low } & 0.691 \\ \text { OC-OD } & 6,1,6,0 & \text { high } & 0.682\end{array}$




\section{Figures and Captions}

Fig. 1. Schematic of a typical, fully connected feedforward neural network.

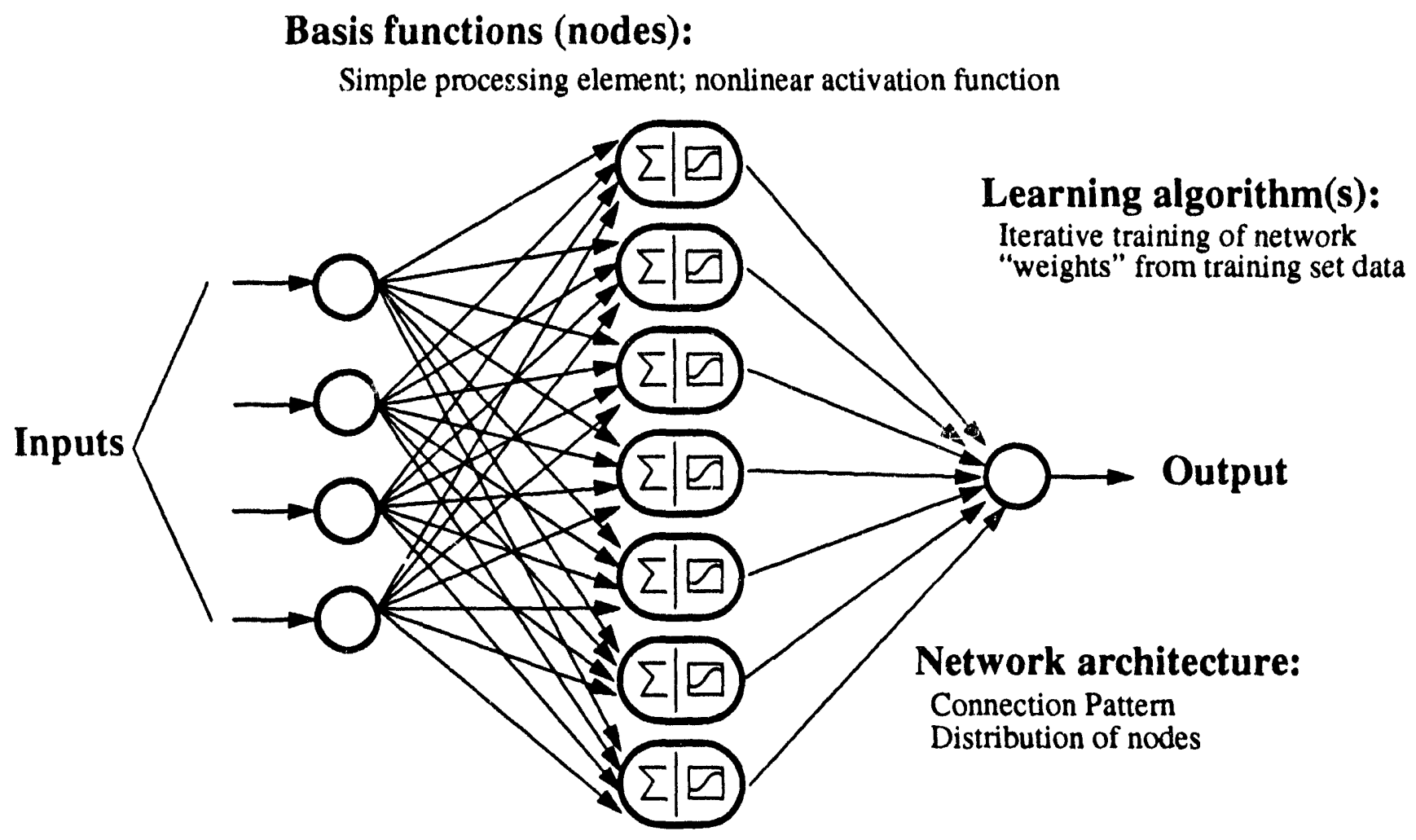

Hidden layer(s) 


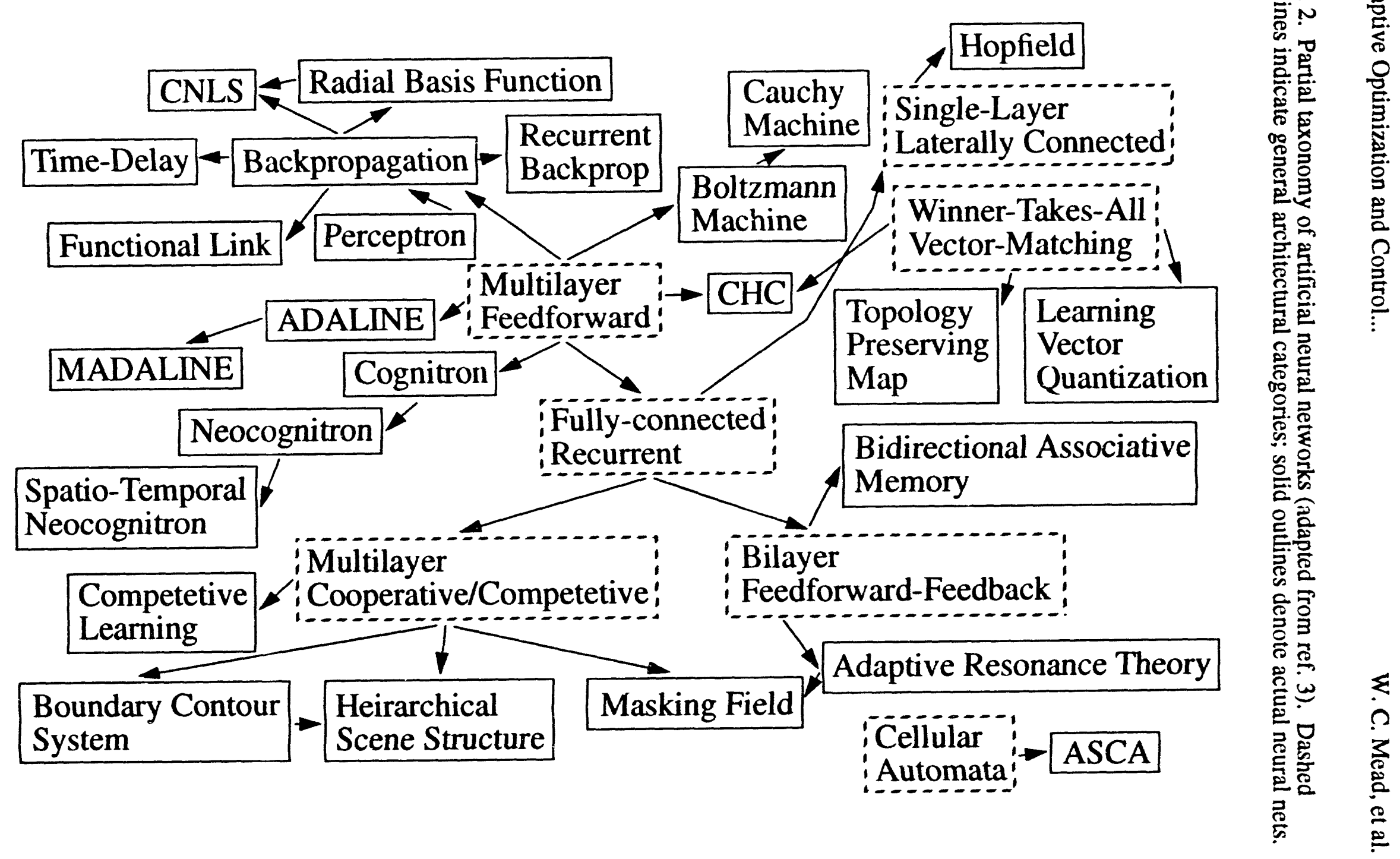


Fig. 3. The backpropagation network uses the sigmoid as a basis function.

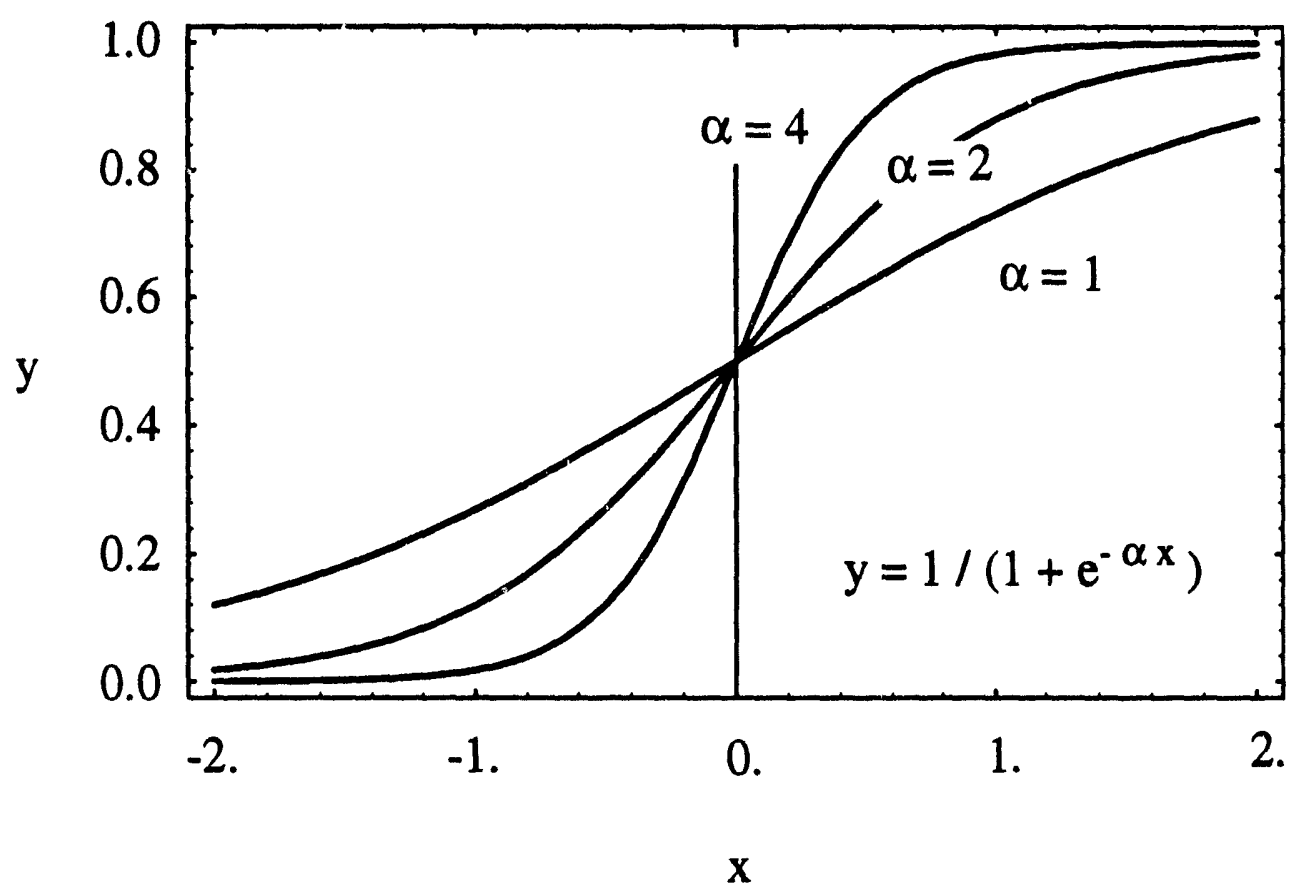


Adaptive Optimization and Control...

W. C. Mead, et al.

Fig. 4. CNLS-network fit to part of the control space of the Free Electron Laser, and the corresponding error surface.
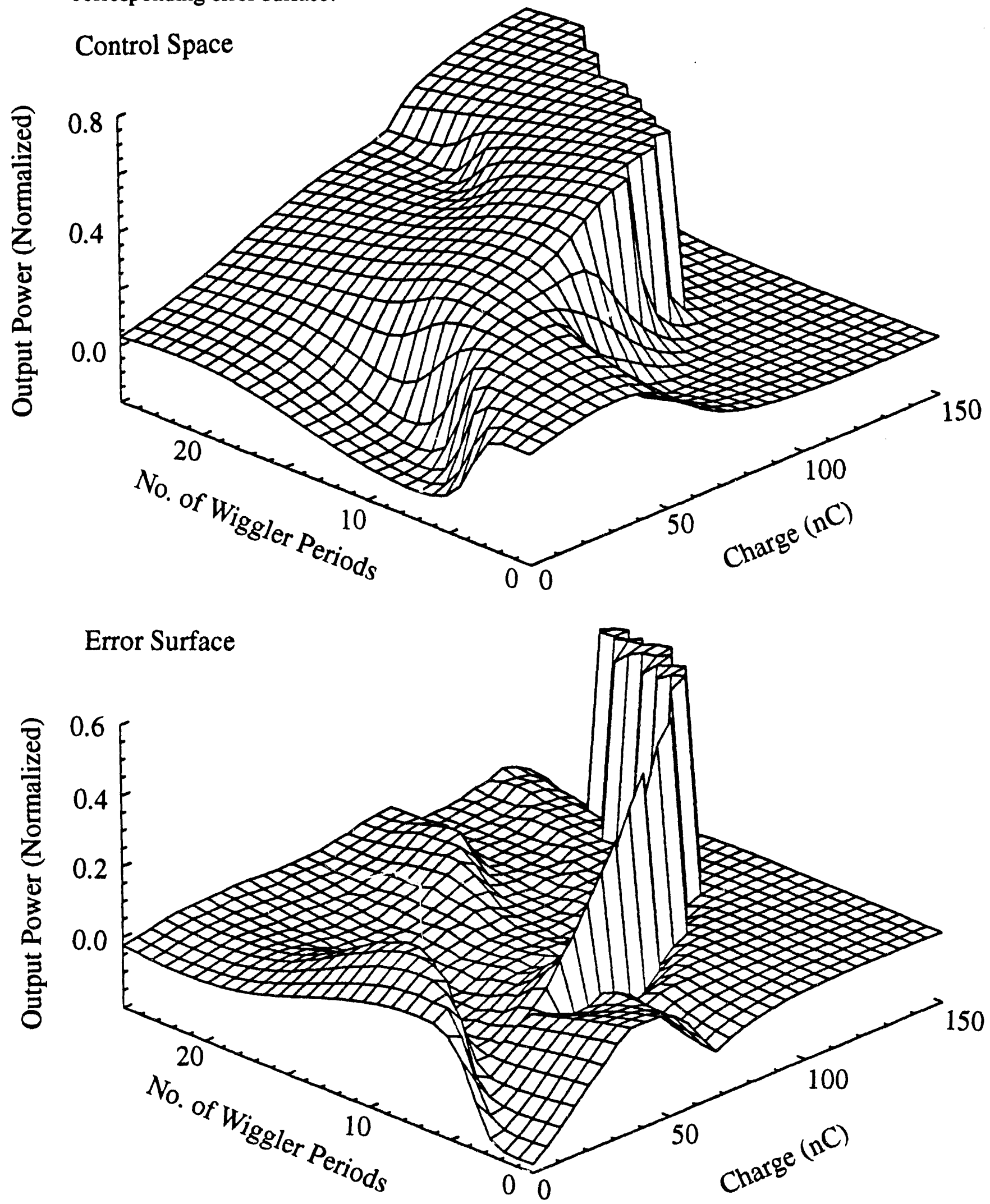
Fig. 5. CNLS-network prediction (dots) compared with tidal data for the Venice lagoon. Network was trained using 1984 data. Predictions shown used input data from previous 12 hours to predict three hours into the future for ten days' 1985 data. Upper threshold shows the 110-cm flood level for activating protective measures.

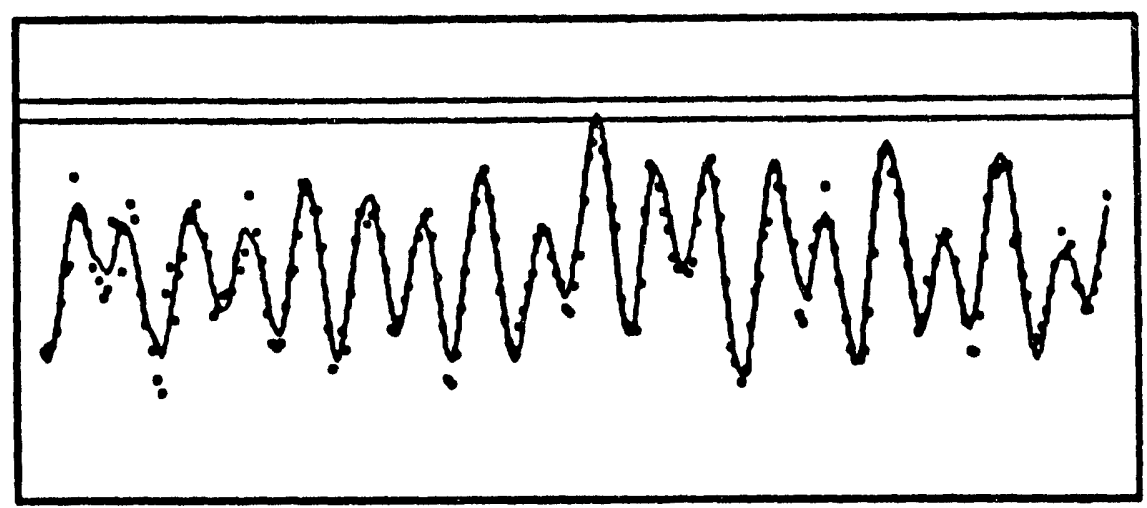


Fig. 6. The negative ion source is compact, with a small active region (from ref. 15). The arc lies in the sub-mm gap between the anode insert and the cathode body. The negative ion beam is extracted by a grid and emerges through a circular aperture located at the top center of this drawing.

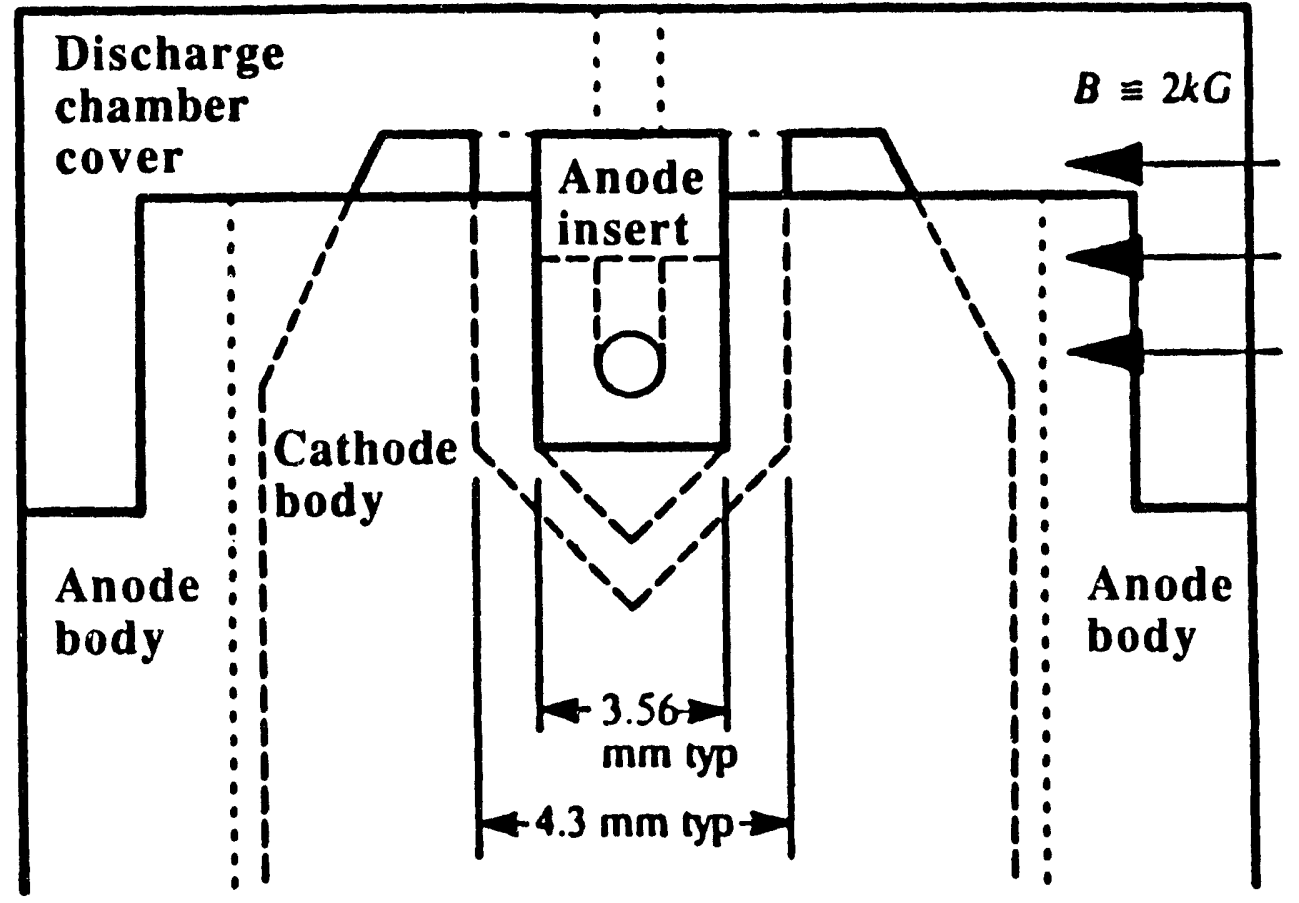


Fig. 7. Schematic of the CTL adaptive control computer code. The CNLS-net artificial neural network $(\mathrm{NN})$ was trained to model unobserved regions of the operating parameter space, based on the datapoints in the operational database.

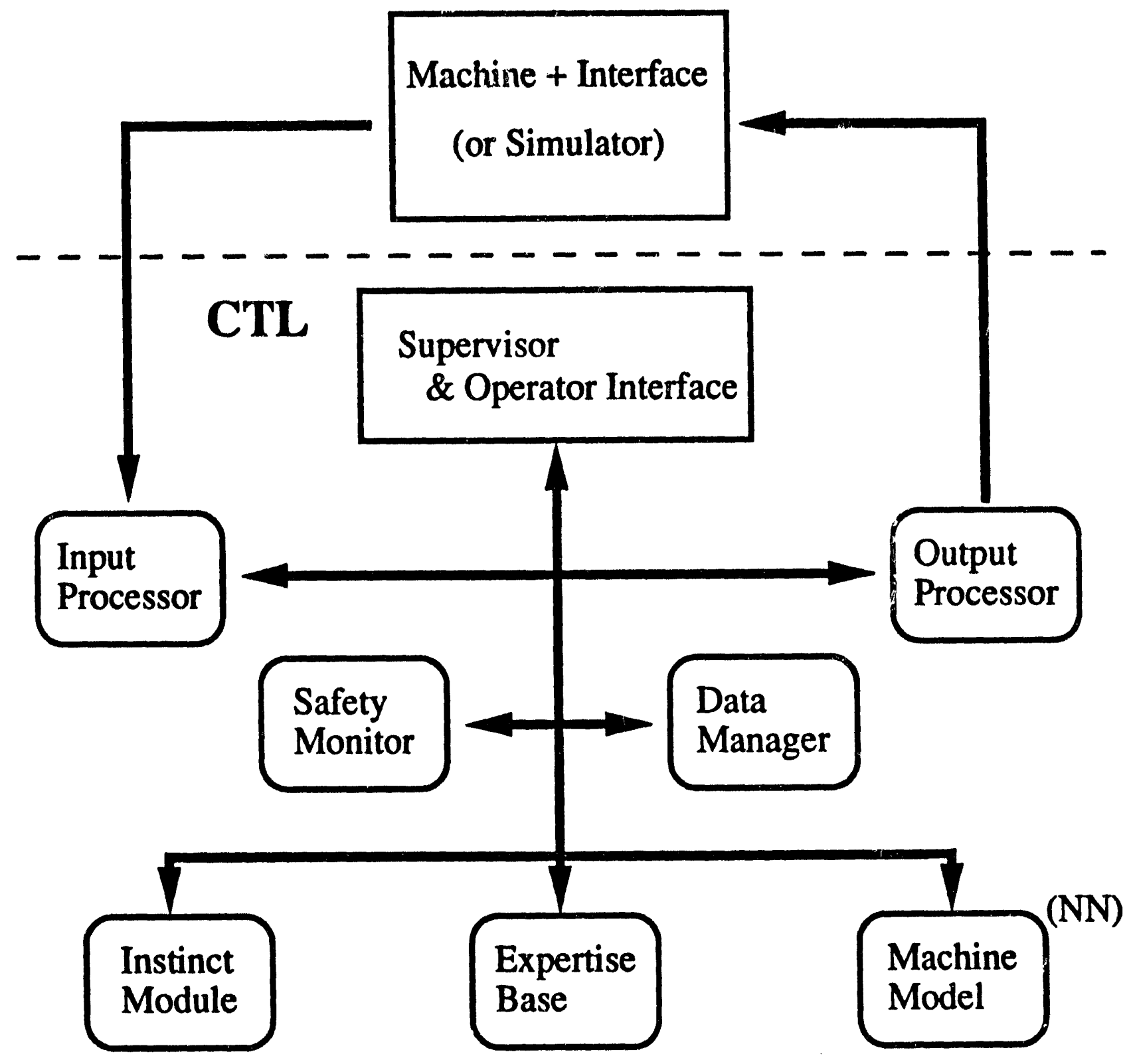


Fig. 8. History of the fourth CTL control run. Four upper panels show control settings chosen by CTL, together with the machine readbacks of the set parameters. Bottom panel shows resulting figure of merit (solid line) and greatest figure of merit achieved as a function of run time. Optimization from "cold" start took about 2 hours. Remainder of run shows beam quality maintenance and rapid recovery from operating interruptions.
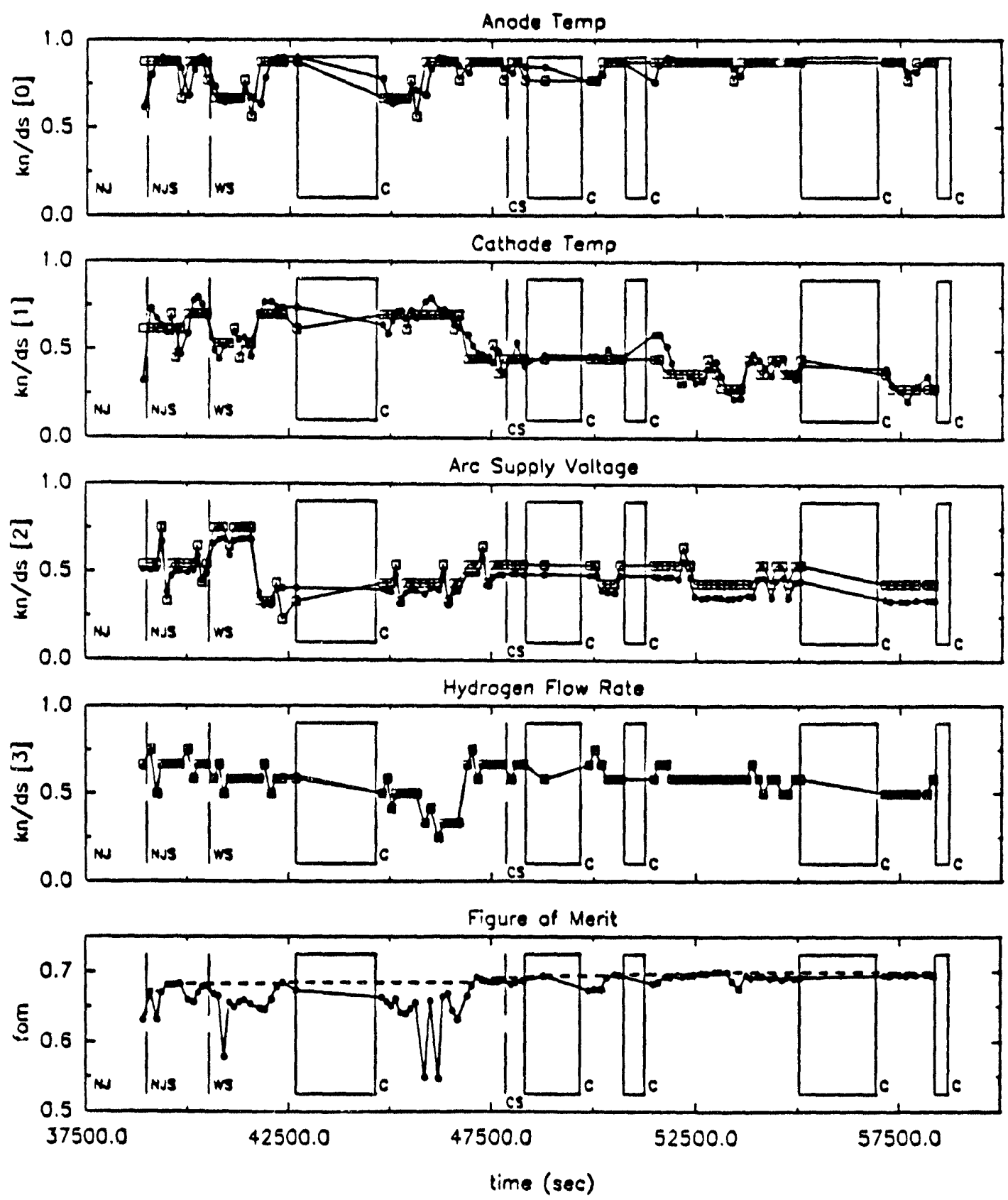
Fig. 9. Superposition of final beam waveforms for six low-impedance control runs. All waveforms show good noise and pulse-to-pulse repeatability. Amplitude is reproducible to within better than $10 \%$.

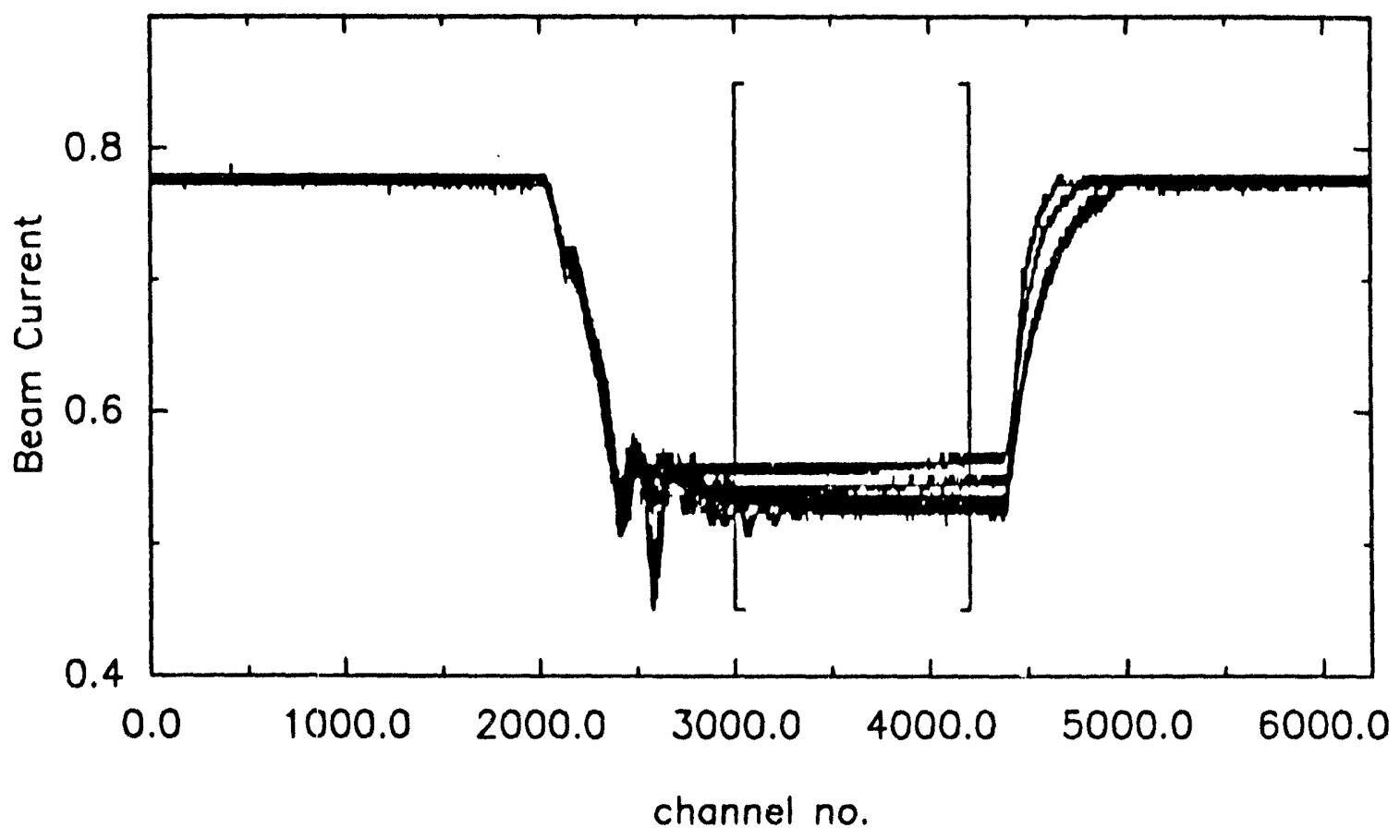




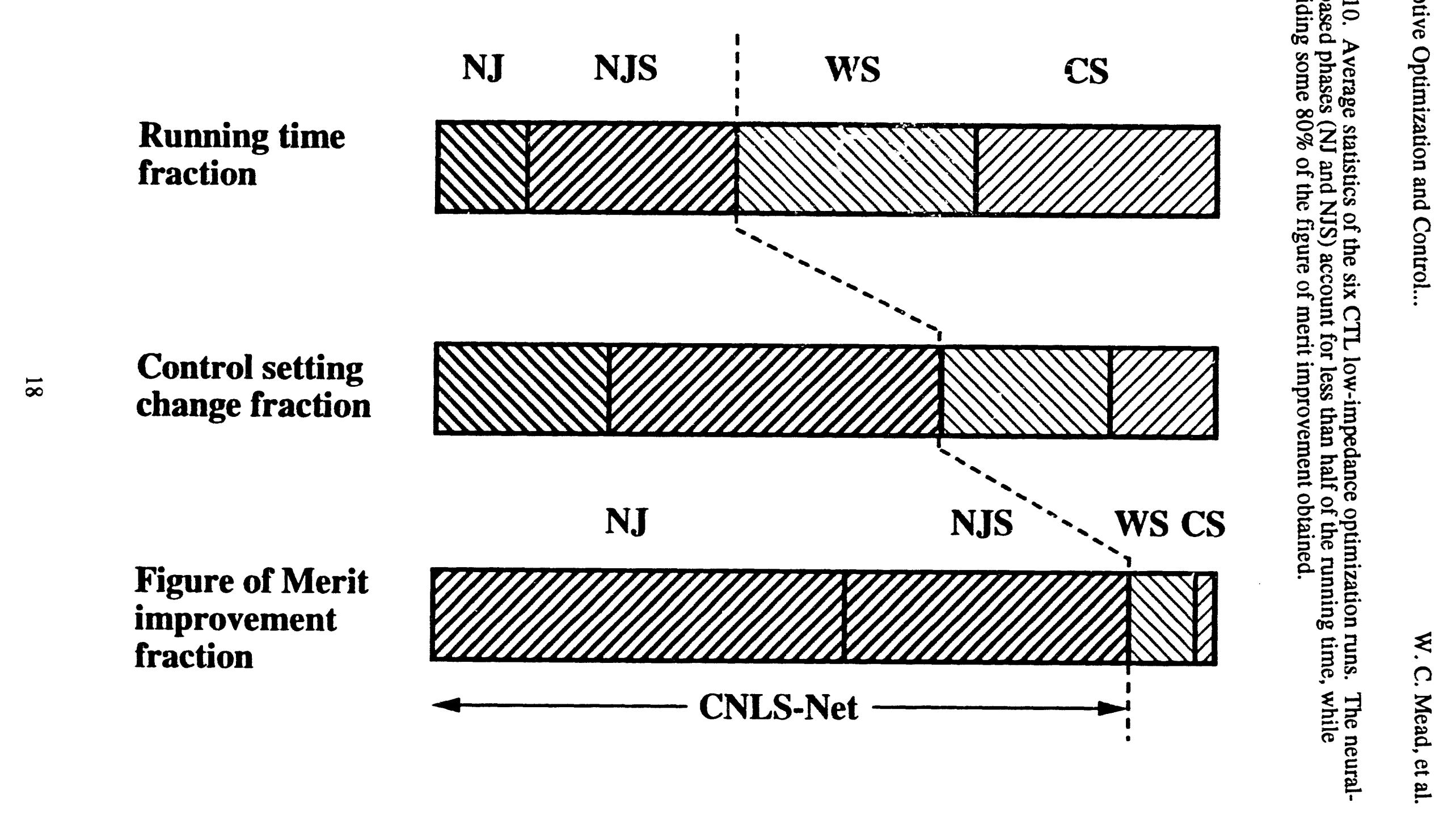



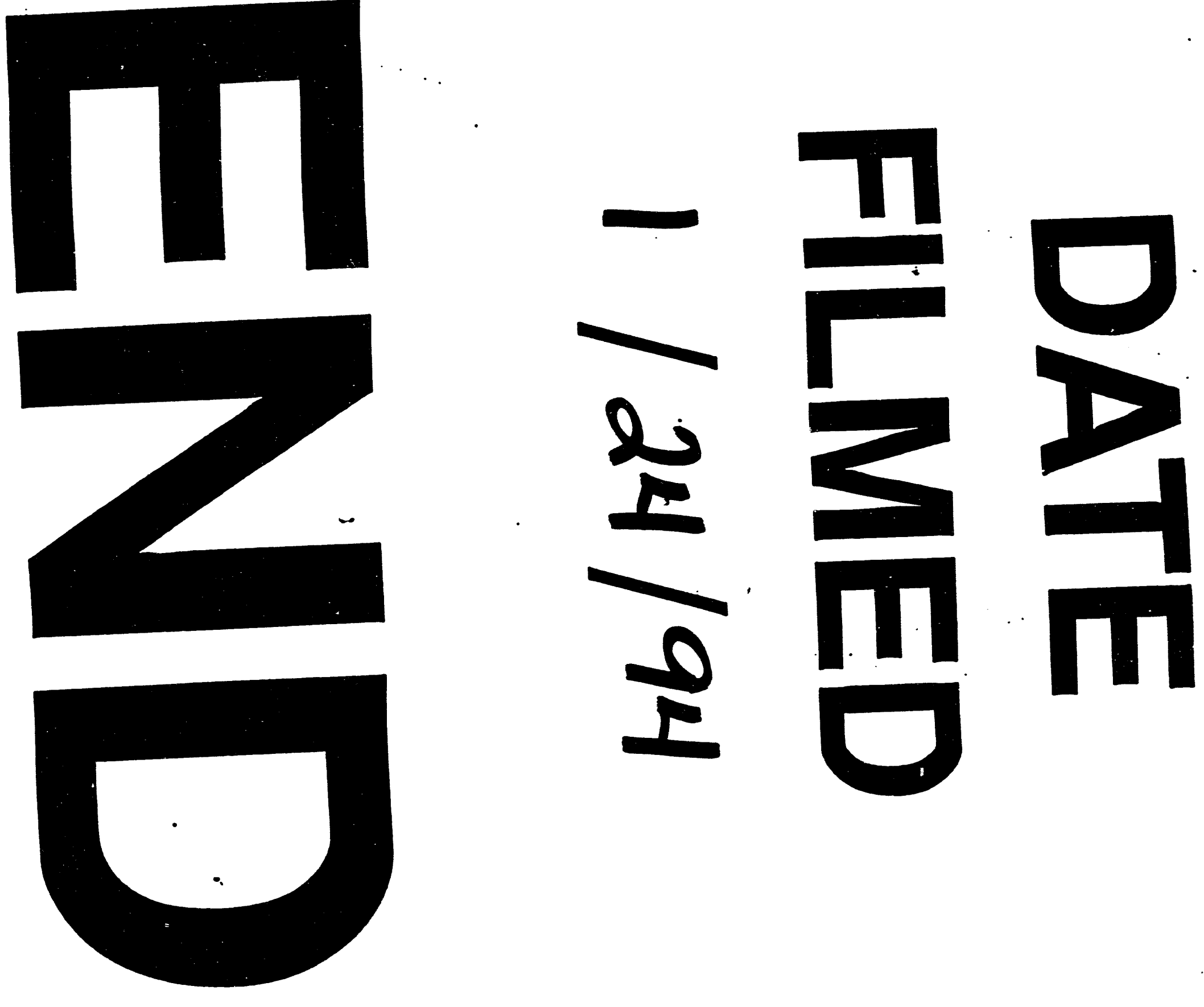


$$
\text { n }
$$

